\title{
The lymphoma-associated NPM-ALK oncogene elicits a p16INK4a/pRb-dependent tumor-suppressive pathway
}

\author{
Paola Martinelli, ${ }^{1}$ Paola Bonetti, ${ }^{1}$ Cristina Sironi, ${ }^{1}$ Giancarlo Pruneri, ${ }^{2}$ Caterina Fumagalli, ${ }^{2}$ Paola Rafaniello Raviele, ${ }^{2}$ \\ Sara Volorio, ${ }^{1}$ Stefano Pileri, ${ }^{3}$ Roberto Chiarle, ${ }^{4}$ Fiona Kate Elizabeth McDuff, ${ }^{5}$ Betsabeh Khoramian Tusi, ${ }^{1}$ \\ Suzanne D. Turner, ${ }^{5}$ Giorgio Inghirami, ${ }^{4}$ Pier Giuseppe Pelicci, ${ }^{1,6}$ and Emanuela Colombo ${ }^{1,6}$ \\ ${ }^{1}$ Department of Experimental Oncology and ${ }^{2}$ Division of Pathology, European Institute of Oncology, Milan, Italy; ${ }^{3}$ Institute of Hematology and Medical Oncology \\ L. e A. Seràgnoli, Policlinico Sant'Orsola-Malpighi, University of Bologna, Bologna, Italy; ${ }^{4}$ Center for Experimental Research and Medical Studies and Department of \\ Biomedical Sciences and Human Oncology, University of Torino, Torino, Italy; ${ }^{5}$ Division of Molecular Histopathology, Department of Pathology, University of \\ Cambridge, Addenbrooke's Hospital, Cambridge, United Kingdom; and ${ }^{6}$ Department of Medicine, Surgery and Dentistry, University of Milan, Milan, Italy
}

\begin{abstract}
Oncogene-induced senescence (OIS) is a barrier for tumor development. Oncogenedependent DNA damage and activation of the ARF/p53 pathway play a central role in OIS and, accordingly, ARF and p53 are frequently mutated in human cancer. A number of leukemia/lymphoma-initiating oncogenes, however, inhibit ARF/p53 and only infrequently select for ARF or p53 mutations, suggesting the involvement of other tumor-suppressive pathways. We report that NPM-ALK, the initiating oncogene of anaplastic large cell lymphomas
\end{abstract}

(ALCLs), induces DNA damage and irreversibly arrests the cell cycle of primary fibroblasts and hematopoietic progenitors. This effect is associated with inhibition of p53 and is caused by activation of the p16INK4a/pRb tumor-suppressive pathway. Analysis of NPM-ALK lymphomagenesis in transgenic mice showed p16INK4a-dependent accumulation of senescent cells in premalignant lesions and decreased tumor latency in the absence of p16INK4a. Accordingly, human ALCLs showed no expression of either p16INK4a or pRb. Up-regulation of the histonedemethylase Jmjd3 and de-methylation at the p16INK4a promoter contributed to the effect of NPM-ALK on p16INK4a, which was transcriptionally regulated. These data demonstrate that p16INK4a/pRb may function as an alternative pathway of oncogene-induced senescence, and suggest that the reactivation of p16INK4a expression might be a novel strategy to restore the senescence program in some tumors. (Blood. 2011;117(24):6617-6626)

\section{Introduction}

Oncogene-induced senescence (OIS) is considered a powerful barrier to the clonal expansion of primary cells carrying mutated oncogenes (like Ras) and a potent tumor-suppressive mechanism in vivo. ${ }^{1}$ Expression of activated oncogenes in primary cells in vitro leads to accumulation of DNA damage and up-regulation of both ARF (alternative reading frame), which stabilizes p53, and $\mathrm{p} 16 \mathrm{INK} 4 \mathrm{a}$, which maintains $\mathrm{pRb}$ (retinoblastoma protein) in its active, hypophosphorylated form. ${ }^{1}$ Notably, high levels of DNA damage, p53, and hypophosphorylated pRb have been documented in various premalignant lesions. ${ }^{2}$

$\mathrm{ARF} / \mathrm{p} 53$ and $\mathrm{p} 16 \mathrm{INK} 4 \mathrm{a} / \mathrm{pRb}$ are the most frequently mutated tumor-suppressive pathways in human tumors. The contribution of each to OIS, however, remains unclear. In primary mouse embryo fibroblasts (MEFs), which have been widely used to model OIS in vitro, its execution depends on ARF and p53 expression, ${ }^{3,4}$ whereas expression of p16INK4a or pRb is dispensable. ${ }^{5,6}$ This suggests that OIS is a DNA damage response mediated by activation of the ARF/p53-pathway. ${ }^{7}$

Although p53 is the most frequently mutated gene in cancer, $\sim 50 \%$ of human cancers do not carry mutations of p53. It is thought that, in this situation, the mutation of other components of the p53 pathway might lead to its functional inactivation. Surprisingly, however, a number of oncogenic proteins lead to functional attenuation of p53 or ARF: PML-RAR $\alpha$ in acute promyelocytic leukemias (APL), ${ }^{8}$ B-cell receptor-ABL in chronic myelogenous leukemias (CML), ${ }^{9}$ NPM-ALK in anaplastic large cell lymphomas (ALCLs $)^{10}$ and cytoplasmic NPM in acute myeloid leukemias (AMLs). ${ }^{11}$ Overall, clinical and experimental evidence suggests that these oncogenes represent the initiating event of tumorigenesis, linking the initial steps of cancer development to their ability to inactivate the ARF/p53 tumor-suppressive pathway. However, considering the relatively low frequency of these tumors in the human population, it is likely that they activate other, p53/ARFindependent, tumor-suppressive pathways.

We investigated the effects of the expression of the oncogene NPM-ALK in primary cells. NPM-ALK, composed of the Nterminal portion of nucleophosmin (NPM) and the intracellular domain of anaplastic lymphoma kinase (ALK), is found in $\sim 80 \%$ of ALCLs. Fusion with NPM activates ALK tyrosine-kinase, leading to the constitutive activation of different downstreampathways, including Ras/ Raf/MEK/ERK, PI3K/AKT, and JAK/ STAT $^{12}-{ }^{13}$ Transgenic expression of NPM-ALK in mice is sufficient to initiate lymphomagenesis in the thymus. ${ }^{14}$ Mutations of p53 are quite rare in NPML-ALK-expressing ALCL $(\sim 5 \%$ $10 \%) .{ }^{15}$ We report here the role of the $\mathrm{p} 16 \mathrm{INK} 4 \mathrm{a} / \mathrm{pRb}$ tumorsuppressor pathway in inhibiting NPM-ALK-dependent tumor progression.
Submitted August 6, 2010; accepted April 4, 2011. Prepublished online as Blood First Edition paper, April 25, 2011. DOI 10.1182/blood-2010-08-301135.

${ }^{\star}$ P.M. and P.B. contributed equally to the manuscript.

The online version of this article contains a data supplement.
The publication costs of this article were defrayed in part by page charge payment. Therefore, and solely to indicate this fact, this article is hereby marked "advertisement" in accordance with 18 USC section 1734. 


\section{Methods}

\section{Cell culture, transfection, infection, and RNA interference}

Primary MEFs, U2OS, and packaging cell lines (Phoenix for retroviral vectors and $239 \mathrm{~T}$ for lentiviral vectors) were grown at $37^{\circ} \mathrm{C}$ in $20 \% \mathrm{O}_{2}$ and $5 \% \mathrm{CO}_{2}$, using DMEM supplemented with $10 \%$ fetal bovine serum. ALCL cell lines (Karpas 299, TS, SU-DHL-1, and JB-6 provided by R.C.; SUP-M2 and DEL provided by S.D.T.) were grown in RPMI plus $10 \%$ serum. Fresh hematopoietic $\mathrm{lin}^{-}$cells were purified from the bone marrow of 8- to 12-week mice according to established procedures ${ }^{16}$ and grown in RPMI plus 10\% serum and cytokines (IL3, IL6, SCF; StemCell Technologies). Transfection was performed with a standard calcium phosphate protocol for Phoenix and 293T cells and with Fugene (Promega) for U2OS cells.

For viral infection, MSCV-based retroviral vectors, expressing GFP together with NPM-ALK, NPM-ALK K210R, mutated H-RasV12, or p16INK4a, were packaged using Phoenix cells and then used to infect primary low-passage MEFs, lin $^{-}$cells, or ALCL cell lines. As a control, an empty MSCV retroviral vector only expressing GFP was used. Successfully infected GFP-positive cells were selected by FACS sorting. MEFs and ALCL cell lines were seeded at low density and counted daily. For colony assays, $5000 \mathrm{lin}^{-}$cells were plated in methylcellulose (MethoCult GF M3434, StemCell Technologies) and colonies were counted after 10 days. For serial replating assays, colonies were collected and disaggregated after counting and the same number of $\operatorname{lin}^{-}$cells was plated again. ${ }^{16}$

For the JunB interference experiments, we used a lentivirus-based vector $\left(\mathrm{PsiCo}^{17}\right)$ expressing the GCATCAAAGTGGAGCGAAA sequence. For NPM-ALK and STAT3 interference in ALCL cell lines (DHL and TS, respectively), we used previously established cells with a doxocyclindependent shRNA inducible system. ${ }^{18}$ Briefly, cells were treated with doxocyclin $1 \mu \mathrm{g} / \mathrm{mL}$ up to 96 hours. At 72 and 96 hours, protein and RNA extracts were prepared and analyzed for p16INK4a levels both by Western blot and Q-PCR (see "qChIP and quantitative PCR analysis" below for details).

\section{Mice}

Experiments involving mice were performed according to national guidelines and after approval by the Institutional Review Board of the European Institute of Oncology. ALK transgenic animals ${ }^{14}$ were bred with p16INK4a $\mathrm{KO}$ mice $^{19}$ and Arf KO mice ${ }^{4}$; all the experiments were conducted with littermate controls.

Thymocyte cell-suspensions were obtained after mechanical disaggregation, filtration ( $40 \mu \mathrm{m}$ cell-strainer), red cell lysis, and PBS washings from the thymi of approximately 6-week-old mice. Cells were permeabilized, stained with an anti Ki67 antibody, and analyzed by FACS.

\section{Immunoblotting and immunofluorescence}

Immunoblotting (WB) and immunofluorescence (IF) experiments were performed as described previously. ${ }^{20}$ All protein lysates for WB were performed in Laemmli buffer. WBs were performed using the following primary antibodies: polyclonal anti-p53 (FL-393, Santa Cruz Biotechnology), polyclonal anti-mouse p16INK4a (M156), antitubulin (all Santa Cruz Biotechnology), anti- $\gamma \mathrm{H} 2 \mathrm{AX}$ (\#613402; Biolegend), polyclonal anti-ARF (Abcam), anti-Jmjd3 antibody (kindly provided by Gioacchino Natoli, European Institute of Oncology, Milan, Italy), polyclonal anti-human p16INK4a (sc368; Santa Cruz Biotechnology), anti-human Rb (554136; BD Biosciences Pharmingen), antiphosphoRb (ser 807/11) (\#9308; Cell Signaling Technology), anti-STAT3 (\#919; Cell Signaling Technology), anti H-RAS (sc29; Santa Cruz Biotechnology).

For IF analysis, cells were seeded on coverslips and 24 hours later fixed in $4 \%$ paraformaldehyde, permeabilized with $0.1 \%$ Triton X-100, and stained with primary mouse anti- $\gamma \mathrm{H} 2 \mathrm{AX}$ antibody (Biolegend) and then with a secondary anti-mouse CY3 fluorochrome-conjugated antibody. Images were acquired at room temperature using a Hamamatsu C4742-95 camera on an Olympus Version 1.0 BX61 microscope. The acquisition software was Cell^F (Olympus).
For the BrdU assay, cells were seeded on coverslips, treated with $33 \mu \mathrm{M}$ BrdU for $15^{\prime}$, fixed with $4 \%$ paraformaldehyde, and analyzed by immunofluorescence using an anti-BrdU antibody (BD Biosciences).

Senescence-associated $\beta$-galactosidase assays were measured in MEFs as described ${ }^{21}$ and in frozen sections of thymuses using the Senescenceassociated $\beta$-galactosidase staining kit (Cell Signaling Technology).

\section{qChIP and quantitative PCR analysis}

For qChIP analyses, MEFs were grown and processed as described ${ }^{22}$ using antibodies against $\mathrm{H} 3 \mathrm{~K} 27_{3 \mathrm{me}}$ (Millipore). The p16INK4a promoter was analyzed in the immunoprecipitated DNA using the primers listed in supplemental Table 1 (available on the Blood Web site; see the Supplemental Materials link at the top of the online article). Immunoprecipitated DNA from $1 \times 10^{6}$ cell equivalents was resuspended in $300 \mu \mathrm{L}$ of $10 \mathrm{mM}$ Tris at $\mathrm{pH}$ 8.0. Real-time PCR was performed with $6 \mu \mathrm{L}$ of DNA per reaction and $200 \mathrm{nM}$ concentration of primers, diluted in a final volume of $20 \mu \mathrm{L}$ of SYBR Green reaction mix (Applied Biosystems). Data represent the mean of 3 measurements \pm SEM.

RNA for expression analysis was extracted using the RNAeasy MiniKit (QIAGEN), treated with DNAse before elution from the column, and retro-transcribed into cDNA for subsequent analysis by quantitative PCR (qPCR). Each PCR contained $10 \mathrm{ng}$ of cDNA template and primers at a concentration of $800 \mathrm{nM}$ in a final volume of $20 \mu \mathrm{L}$ of SYBR Green reaction mix. Data represent the mean of 3 measurements \pm SEM. Primers are listed in supplemental Table 1 .

\section{Luciferase assay}

U2OS cells and MEFs were transfected with pCDNA3-based vectors expressing NPM-ALK, NPM-ALK K210R, or mutated H-Ras, together with a luciferase-based synthetic reporter for $\mathrm{p} 53$ activity ${ }^{20}$ and a cytomegalovirus-LacZ vector as transfection control. Control U2OS cells and MEFs were transfected with a pCDNA3 vector together with the same luciferasebased synthetic reporter for p53 activity ${ }^{20}$ and cytomegalovirus-LacZ vector. Luciferase activity was measured with a luminometer, using a commercial luciferin solution (Promega) as a substrate. Results were normalized for transfection efficiency by measuring the $\beta$-galactosidase activity.

\section{DNA extraction, bisulfite treatment, and methylation-specific PCR (MSP)}

Genomic DNA was isolated from formalin-fixed paraffin-embedded samples, using a commercial kit (QIAamp DNA FFPE Tissue kit, QIAGEN) according to the manufacturer's instructions. In each case, the percentage of neoplastic cells was assessed in hematoxylin and eosin-stained slides obtained from the same blocks before DNA isolation: the mean percentage was 70\% (range 50\%-80\%). Sodium bisulfite modification was performed using EZ DNA Methylation Kit (Zymo Research). PCR primers distinguishing between methylated and unmethylated p16 were used ${ }^{23}$ (supplemental Table 1). Each run of amplification included (i) commercially available CpG dinucleotide-completely methylated DNA (CpGenome Universal Methylated DNA; Chemicon-Millipore) as a positive control; (ii) 2 different negative controls, either $\mathrm{CpG}$ dinucleotide completely unmethylated DNA (CpGenome Universal Unmethylated DNA; Chemicon-Millipore), or DNA untreated by sodium bisulfite. The PCR products underwent electrophoresis on $2 \%$ agarose gel and were then visualized under ultraviolet illumination using ethidium bromide staining. Two methylation-specific PCR (MSP) experiments for each sample were performed.

\section{Tp53 gene sequencing}

Genomic DNA was isolated from formalin-fixed paraffin-embedded samples and used for PCR amplification. We designed primers (listed in supplemental Table 1) to amplify the $t p 53$ gene from exon 5 to exon 8 under the same PCR condition: 40 cycles, 94 degrees for 30 seconds, 60 degrees for 45 seconds, 72 degrees for 45 seconds. The PCR fragments were run on a $2 \%$ agarose gel, then quantified and purified with EXO-SAP enzymes (Life Sciences). The purified amplicons were sequenced by using a BD 3.1 cycle 
sequencing kit (Applied Biosystems) and a 3730xl Sequencer, in standard conditions. The results were analyzed using Mutation Surveyor 4.0 software.

\section{Immunohistochemical analysis}

The use of patient samples was approved by our Institutional Review Board; all patients gave informed consent according to the Declaration of Helsinki. Immunostaining for $\mathrm{pRb}, \mathrm{p} 16 \mathrm{INK} 4 \mathrm{a}$, and $\mathrm{p} 53$ was performed on $3-\mu \mathrm{m}$-thick formalin-fixed, paraffin-embedded sections after heat-induced antigen retrieval using polyclonal antisera (pRb, Santa Cruz Biotechnology) or the monoclonal anti-p53 antibody DO7 (Dako) and anti-p16INK4a (E6H4; CINtec). At least 500 neoplastic cells at $\times 400$ magnification were analyzed by a qualified hematopathologist (Dr Pruneri, Division of
Pathology, European Institute of Oncology, Milan, Italy). Control sections for antibody specificity included (i) sections from known positive control tissues (for $\mathrm{pRb}$ : colon cancer; for p53: breast cancer bearing a p53 missense mutation leading to an intense nuclear immunoreactivity in more than $90 \%$ of the neoplastic cells; for p16INK4a: squamous cell carcinoma of the cervix); (ii) serial sections of the cases tested stained with an unrelated isotypic mouse monoclonal antibody (for p16INK4a and p53) and normal rabbit serum (for $\mathrm{pRb}$ ), as negative controls.

\section{Statistics}

Statistical analysis was performed using JMP Version 6.0 software. Statistical significance was evaluated by nonparametric Mann-Whitney U
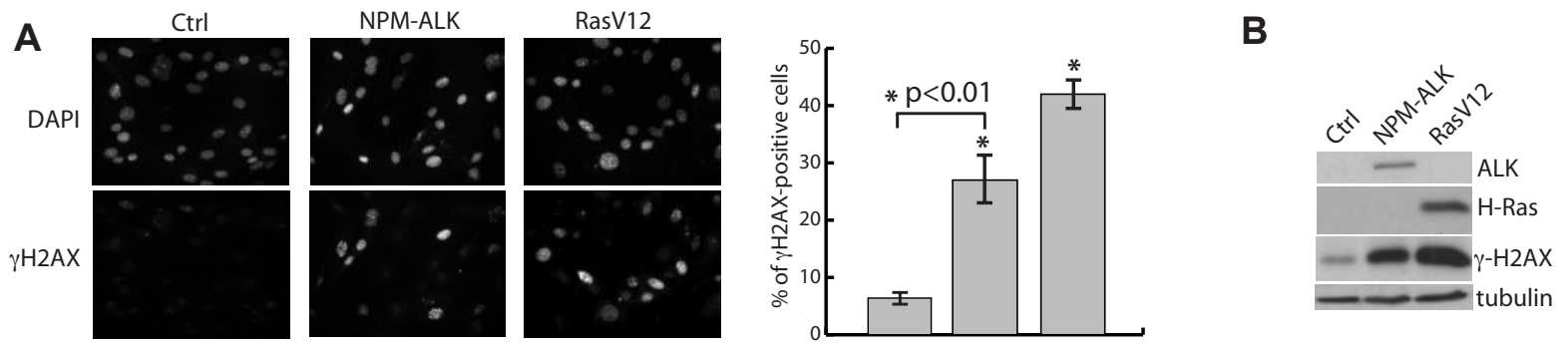

D
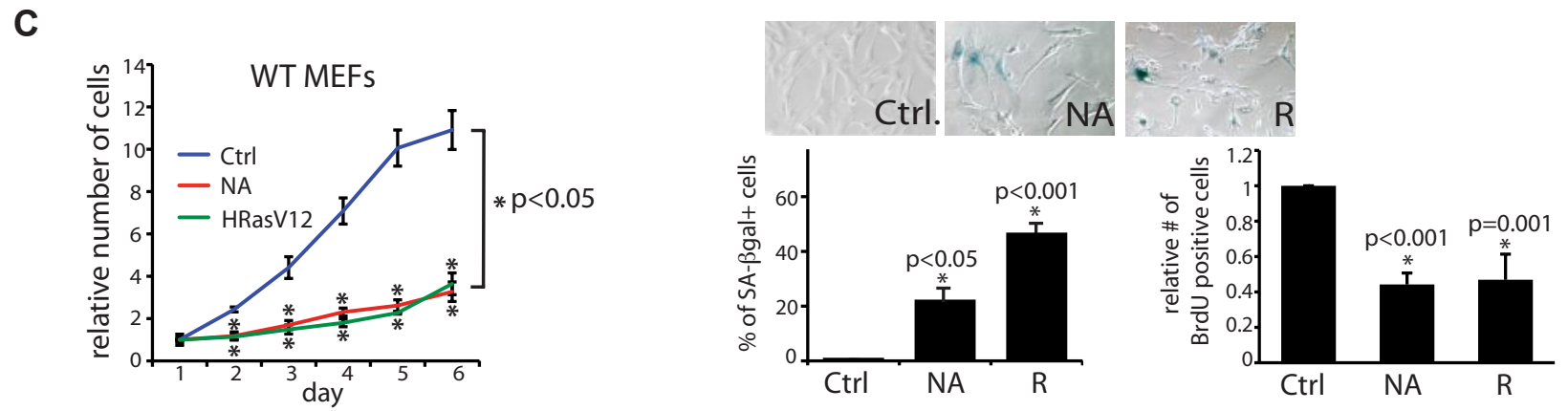

E

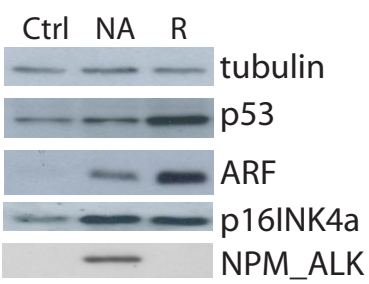

$\mathbf{F}$

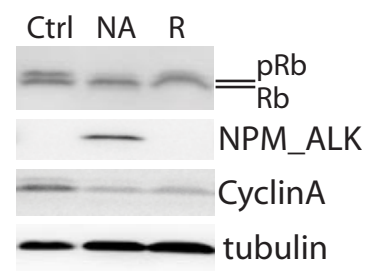

G

$\mathbf{H}$
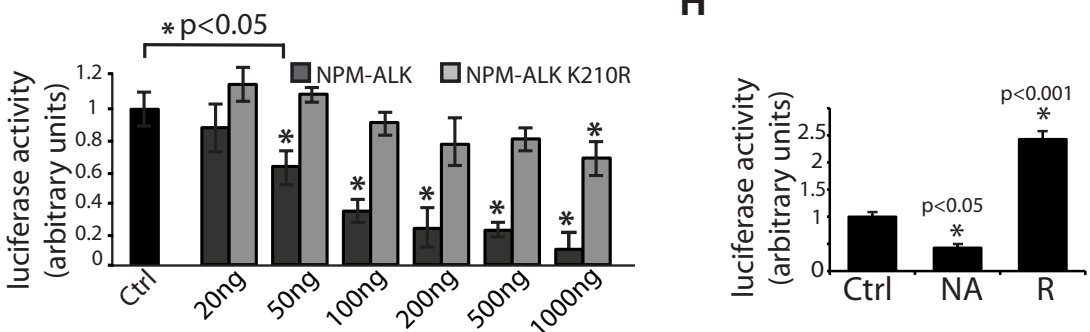

Figure 1. NPM-ALK inhibits p53 activity and it induces a senescent checkpoint in primary fibroblasts. (A-F) WT MEFs were infected with control (Ctrl) and NPM-ALK (NA) or RASV12 expressing retroviruses. (A) Cells were analyzed (5 days after infection) by immunofluorescence (magnification $\times 600)$ and Western blot (B) with an anti- $\gamma \mathrm{H} 2 \mathrm{AX}$ antibody (representative micrographs and percentage of labeled cells are shown). (C) Growth curve of infected cells plated after infection and counted daily (cell numbers are expressed relative to day $1 ;{ }^{*} P<.05$ relative to control cells infected with an empty vector). (D) $\beta$-galactosidase detection (representative micrographs [magnification $\times 600$ ] and percentage of positive cells) and anti-BrdU staining (percentage of positive cells relative to control cells) performed 6 and 4 days after infection, respectively. (E) Western blotting of p53, ARF, p16INK4a, NPM-ALK, and tubulin expression, 4 days after infection. (F) Western blotting of pRb, NPM-ALK, cyclin A, and tubulin expression, 3 days after infection. (G) Luciferase assay in WT MEFs transduced with the pGL13 p53-reporter, the CMV- $\beta$ Gal vector, and increasing amounts of vectors expressing NPM-ALK or the K210R kinase-dead NPM-ALK mutant (or a control empty vector). Data are from 3 independent experiments and normalized to $\beta$ Gal. ${ }^{*} P<.05$ relative to control cells infected with an empty vector. (H) Same Luciferase assay as in panel G comparing a single dose (100 ng) of NPM-ALK- and RASV12-expressing vectors. 

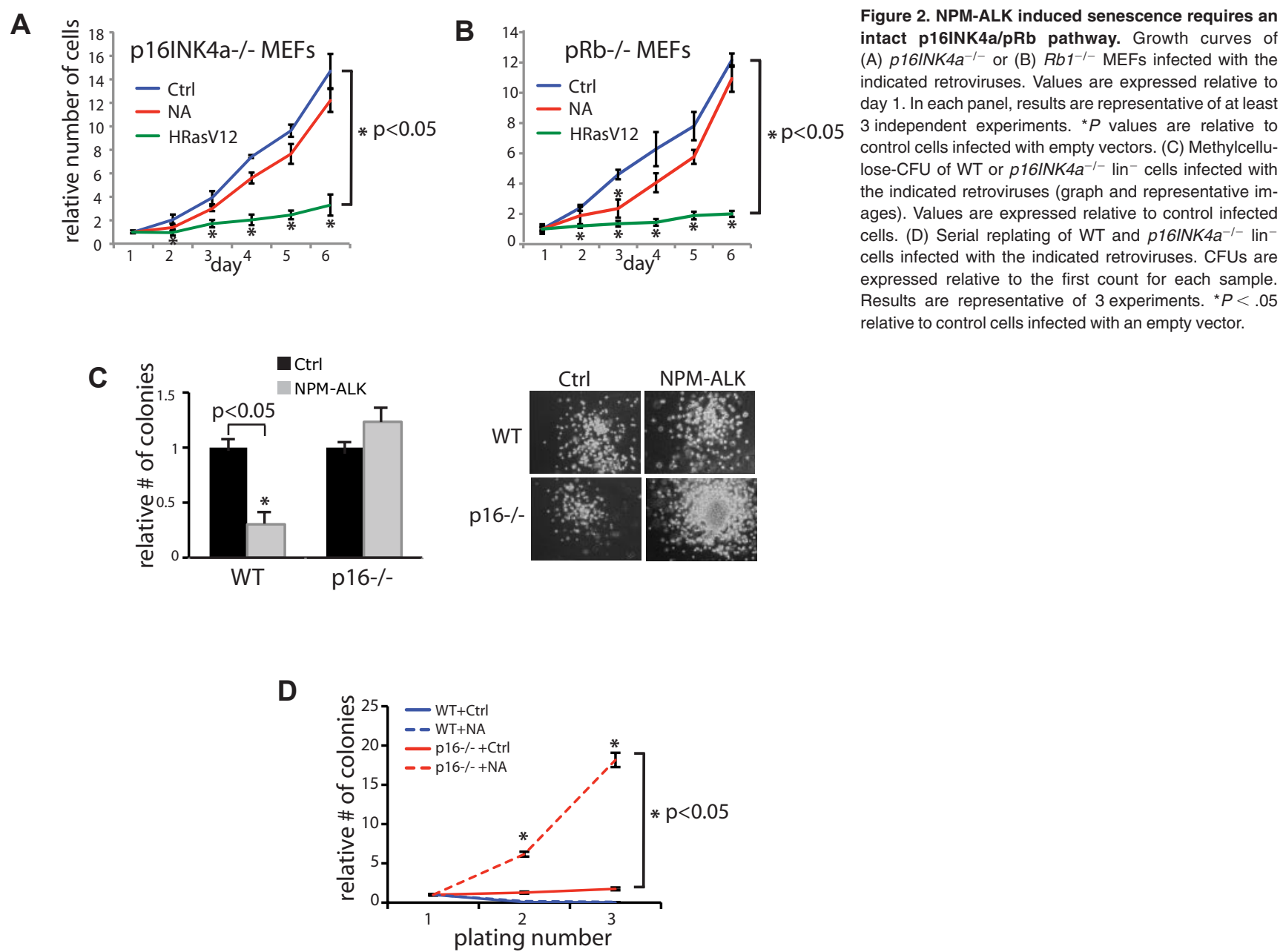

test (Wilcoxon-Kruskal Wallis). Mouse survival was analyzed using the Kaplan-Meier survival curve.

\section{Results}

Early-passage wild-type (WT) MEFs were infected with retroviruses coding for the green fluorescence protein (GFP) and either NPM-ALK (NA vector) or oncogenic RAS (Valine-12 mutation; RasV12 vector) proteins. Cells were infected for 2 days, replated, and analyzed at different time points. Activation of a DNA-damage response was assessed by immunostaining and Western blotting of phosphorylated H2AX ( $\gamma \mathrm{H} 2 \mathrm{AX})$. Like RasV12, the expression of NPM-ALK caused accumulation of $\gamma \mathrm{H} 2 \mathrm{AX}$ in the cells (Figure 1A-B). Analysis of the effects of NPM-ALK or RasV12 on cell proliferation showed inhibition of fibroblast growth with both oncogenes (Figure 1C). Notably, NPM-ALK and RasV12expressing cells became large and flat, acquired positivity for the SA- $\beta$-galactosidase senescence-associated marker $(\sim 20 \%$ and $\sim 50 \%$ of cells, respectively), and showed reduction of bromodeoxyuridine (BrdU) incorporation (Figure 1D). The growthinhibitory effect of NPM-ALK was dependent on an intact kinase domain (supplemental Figure 1). Together, these findings suggest that the expression of the NPM-ALK kinase in primary fibroblasts, like that of oncogenic Ras, induces DNA damage and cellular senescence.

To investigate the molecular basis of NPM-ALK-induced senescence, we analyzed the protein levels of p53, ARF, and
p16INK4a by Western blotting. As expected, RasV12 induced marked up-regulation of p16INK4a, ARF, and p53 (Figure 1E). Accordingly, it activated reporter gene transcription from a synthetic p53-dependent promoter in the same cells (Figure 1H). Like RasV12, NPM-ALK induced p16INK4a expression, whereas it only modestly increased levels of p53 and ARF (Figure 1E) and reduced p53-dependent transcription in our experimental system (Figure 1G-H). Again, the activity relied on an intact kinase domain (Figure 1G). Thus, at variance with RasV12, which activates both p53 and p16INK4a, NPM-ALK activates p16INK4a and, as previously reported, inhibits p53 transcriptional activity. ${ }^{10}$ P16INK4a inhibits the activity of cyclin dependent kinases (CDKs) that in turn phosphorylate $\mathrm{pRb}$, blocking its activity. Therefore, we evaluated levels of pRb phosphorylation. As shown in Figure 1F, NPM-ALK- and RasV12-infected MEFs showed reduced levels of $\mathrm{pRb}$ phosphorylation (which correlates with the observed cellcycle arrest and low S-phase specific cyclin A), thus confirming increased p16INK4a activity in these cells.

To investigate whether activation of the $\mathrm{p} 16 \mathrm{INK} 4 \mathrm{a} / \mathrm{Rb}$ pathway is functionally required for NPM-ALK-induced senescence of fibroblasts, NPM-ALK (or RasV12) was expressed in MEFs derived from p16INK4a- or Rbl-null mice. RasV12 expression inhibited proliferation in both cell types, confirming that this effect is $\mathrm{p} 16 \mathrm{INK} 4 \mathrm{a} / \mathrm{pRb}$-independent, as already reported. ${ }^{5,6}$ Conversely, NPM-ALK failed to induce a proliferative block in either $p 16 I N K 4 a$ or Rb1-null MEFs (Figure 2A-B), suggesting that NPM-ALK- 

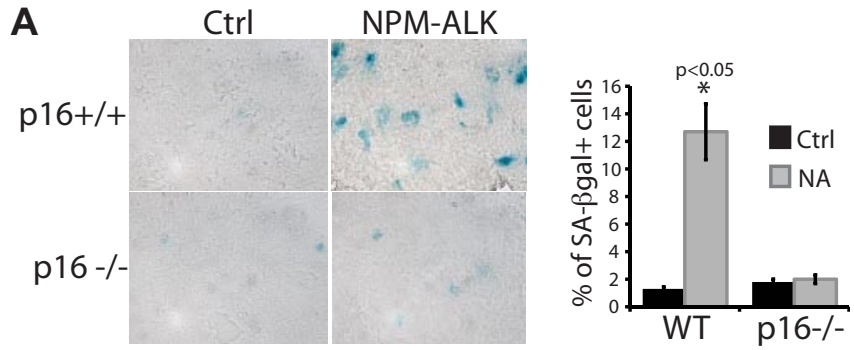

B
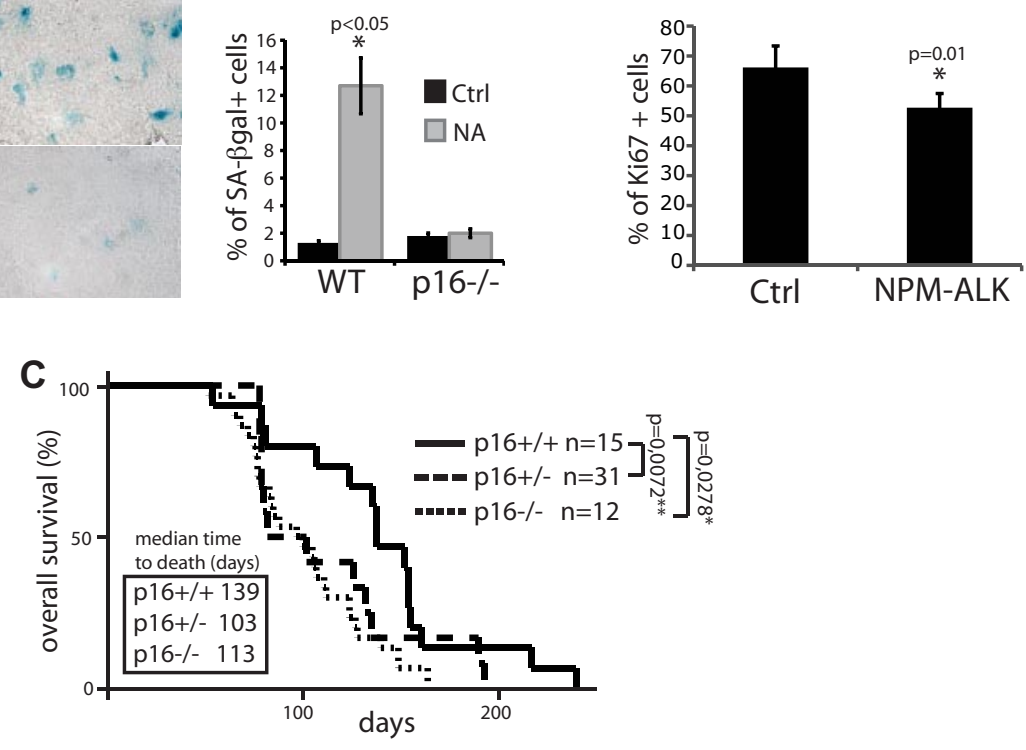

D

W: $16+/+\quad H: p 16+/-\quad K: p 16-/-$

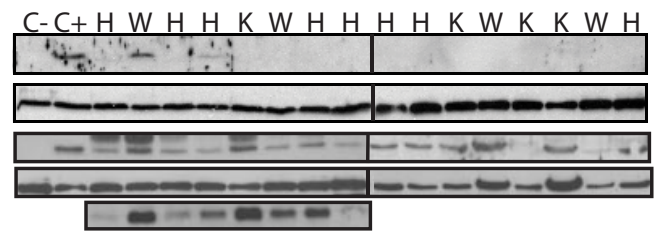

p16INK4a
tubulin
p53
vinculin
p19ARF

Figure 3. NPM-ALK activates the p16INK4a pathway in vivo. (A) SA- $\beta$-gal staining of frozen sections from 1.5-month-old mice of the indicated genotypes (representative images [magnification $\times 400$ ] and percentage of positive cells). (B) Percentage of Ki67-positive cells from thymocyte cell-suspensions of 1.5-month-old NPM-ALK transgenic or control littermates, as evaluated by FACS analysis. Results were obtained from 3 independent experiments, each performed using at least 2 mice of each genotype. (C) Survival curves of NPM-ALK transgenic mice of different genotypes for p16INK4a, as indicated. Median time to death is indicated in the inset. (D) Western blot analyses of p16INK4a, p53, and ARF expression in tumors derived from NPM-ALK transgenic mice of different p16INK4a genotypes, as indicated. Tubulin or vinculin was used as loading control. C+ indicates WT MEFs (positive control); C-, p16INK4a- or Tp53-null MEFs (negative controls).

induced senescence of primary fibroblasts is, instead, p16INK4a/pRb-dependent.

To confirm these findings into another type of primary cells, NPM-ALK was expressed into a bone marrow subpopulation enriched in stem cells and progenitors (lineage negative [ lin $\left.^{-}\right]$ cells). These cells likely contain putative lymphoma-initiating cells, as shown by the findings that transplantation of NPM-ALKtransduced bone marrow ${ }^{24}$ or lin $^{-}$(data not shown) cells results in lymphoma development in the recipient mice. Expression of NPM-ALK in lin $^{-}$cells induced a marked reduction in their proliferation properties, which was completely rescued by the loss of pl6INK4a (as assessed by the methyl-cellulose colonyformation assay; Figure 2C). Notably, the colonies obtained from p16INK4a-null $\mathrm{lin}^{-}$cells expressing NPM-ALK were markedly larger (Figure 2C). Control primary colonies from WT and p16INK4a-null $\mathrm{lin}^{-}$cells exhausted their proliferative potential after the third replating in methylcellulose (Figure 2D). Strikingly, the number of NPM-ALK-expressing p16INK4a-null colonies, but not of the NPM-ALK-expressing WT colonies, expanded geometrically at each replating (Figure 2D). Together, these observations demonstrate that loss of $p 16 I N K 4 a$ prevents the proliferative arrest induced by NPM-ALK in hematopoietic progenitors and unmasks its oncogenic potential, as shown by the increased clonogenic activity (numbers of primary colonies), proliferative activity (colony size), and replication potential (serial replating).
Finally, to assess the in vivo role of p16INK4a in an NPM-ALKmediated lymphomagenesis model, we used mice expressing the NPM-ALK transgene under the control of the lymphoid-specific CD4 promoter; these mice develop thymic lymphomas with a mean survival of 18 weeks. ${ }^{14}$ Thymi from NPM-ALK transgenic mice (collected before the occurrence of histopathologic signs of lymphoma) showed a significantly higher frequency of SA- $\beta$ galactosidase-positive cells ( $10 \%-15 \%$ vs $0.5 \%-1.5 \%$; Figure 3A), and a reduction in Ki67-positive cells (Figure 3B), compared with matched control littermates. Strikingly, thymi from NPMALK-p16INK4a-null intercrosses showed frequencies of senescence-like cells comparable to that of WT or p16INK4a-null mice (Figure 3A), suggesting that NPM-ALK-induced senescence in vivo also depends on p16INK4a expression.

To examine in vivo the effect of the $\mathrm{p} 16 \mathrm{INK} 4 \mathrm{a} / \mathrm{pRb}$ pathway in limiting tumor progression, we monitored NPM-ALK tumorigenesis in a p16INK4a-null or p16INK4a-heterozygous (p16INK4ahet) background. All the analyzed NPM-ALK transgenic mice, regardless of their p16INK4a genotype, died as a consequence of thymic lymphomas (with large or medium-sized cells; not shown). Tumor latency in the transgenic mice expressing NPM-ALK in a p16INK4a-WT context was as reported. ${ }^{14}$ Loss of either one or both p16INK $4 a$ alleles resulted in a significant reduction in tumor latency, with a mean time-to-death of 103 and 113 days in the 
Table 1. Expression of p16INK4a, p53, and pRb in 19 tumor specimens from ALCL ALK ${ }^{+}$patients by immunohistochemical analysis with specific antibodies

\begin{tabular}{|c|c|c|c|c|c|}
\hline samples & p16INK4a & pRb & p53 & $\begin{array}{c}\text { p16- } \\
\text { promoter }\end{array}$ & p53-seq \\
\hline 1 & 0 & 0 & 4 & UNMET & WT \\
\hline 2 & 5 & 0 & 20 & MET & nt \\
\hline 3 & 6 & 0 & 20 & UNMET & WT \\
\hline 4 & 0 & 0 & 15 & MET & WT \\
\hline 5 & 0 & 0 & 25 & UNMET & WT \\
\hline 6 & 7 & 0 & 9 & nt & nt \\
\hline 7 & 0 & 0 & 3 & nt & WT \\
\hline 8 & 50 & 0 & 2 & UNMET & WT \\
\hline 9 & 5 & 0 & 30 & UNMET & WT \\
\hline 10 & 3 & 2 & 20 & nt & WT \\
\hline 11 & 0 & 0 & 15 & nt & WT \\
\hline 12 & 45 & 30 & 70 & nt & WT \\
\hline 13 & 0 & 0 & 2 & UNMET & WT \\
\hline 14 & 0 & 20 & 7 & MET & WT \\
\hline 15 & 60 & 0 & 5 & UNMET & nt \\
\hline 16 & 10 & 0 & 3 & nt & nt \\
\hline 17 & 0 & 0 & 12 & nt & nt \\
\hline 18 & 3 & 0 & 4 & UNMET & WT \\
\hline 19 & 0 & 0 & 17 & nt & nt \\
\hline
\end{tabular}

Negative samples are highlighted in green. Numbers represent the percentage of positive tumor cells in each sample. Analyses of Tp53 gene sequence (p53seq) and p16INK4a promoter methylation state (p16-promoter) are also included.

p16INK4a-het and p16INK4a-null backgrounds, respectively, compared with 139 days in the WT background (Figure 3C). The observation that tumor latency was similarly reduced in the p16INK4a-het and p16INK4a-null mice suggests the existence of a strong pressure to lose or inactivate pl6INK4a during ALCL tumorigenesis. Indeed, we did not observe any detectable p16INK4a expression in 7 of 8 lymphomas obtained from p16INK $4 a$-het mice, nor in 3 of 4 tumors from WT mice (Figure 3D). Notably, the same samples showed expression of p53 and ARF (Figure 3D). These data indicate that NPM-ALK-driven tumorigenesis in mice is physiologically constrained by the activation of the p16INK4a tumor-suppressor program, and that its inactivation is necessary for ALCL to develop.

Having shown, both in vitro and in vivo, that the expression of NPM-ALK elicits a senescence response, that in turn limits cell proliferation in vitro and tumor progression in vivo, the next step was to verify whether loss of p16INK4a and/or pRb expression is a necessary requisite for tumor development. We performed this analysis in human NPM-ALK-expressing ALCL samples, using primary patient samples and established cell lines. Expression of p16INK4a and $\mathrm{pRb}$ in primary ALCLs was evaluated by immunohistochemistry in 19 samples (Table 1 and supplemental Figure 2 for representative results). Notably, 17 showed no expression of either the $\mathrm{p} 16 \mathrm{INK} 4 \mathrm{a}$ or $\mathrm{pRb}$ protein, 1 sample very low expression of both proteins, and 1 sample a high percentage of tumor cells expressing both $\mathrm{p} 16 \mathrm{INK} 4 \mathrm{a}$ and $\mathrm{pRb}$. The $\mathrm{p} 16 \mathrm{INK} 4 \mathrm{a}$ promoter was hypermethylated in 3 of 11 cases analyzed (see supplemental Figure 3 for representative results). Of these 3 cases, 2 displayed no p16INK4a expression and 1 very few (5\%) p16INK4a-expressing cells. Several samples (10/19) showed a medium to high percentage of p53 positive tumor cells (between $11 \%$ and $70 \%$ ), the remaining samples displayed low positivity (between $2 \%$ and $10 \%$; Table 1). We also performed mutational analysis of the Tp53 gene. Successful DNA amplification of exons 5 to 8 was obtained in 13 cases, all of them showing a WT sequence.
We then evaluated the expression of p16INKa and $\mathrm{pRb}$ in 6 ALCL cell lines by Western blot analysis. Three showed no expression of p16INK4a (Figure 4A) and hypermethylation of its promoter (see supplemental Figure 3 for representative results). All 6 cell lines showed pRb expression (Figure 4A). Notably, and regardless of the expression of p16INK4a, all of them showed high levels of the phosphorylated form of $\mathrm{pRb}$, which corresponds to its inactive state (Figure 4A). Thus, other mechanisms may have been selected in the p16INKa-expressing ALCL cell lines to inactivate $\mathrm{pRb}$. Together, these data suggest that inactivation of either p16INK4a or pRb is a near-constant feature of human NPM-ALKexpressing ALCLs. Their relative frequency, however, was significantly different in primary samples versus cell lines. In particular, $\mathrm{pRb}$-positive ALCL cases were very frequent in the ALCL cell lines, suggesting that expression of hyperphosphorylated $\mathrm{pRb}$ might be critical for growth in culture and establishment of a cell line. This observation correlates with the fact that the $\mathrm{pRb}$-positive ALCLs express hyperphosphorylated $\mathrm{pRb}$, are more aggressive, and are associated with a worse prognosis. ${ }^{25}$

We then investigated the mechanistic relations among NPMALK expression, p16INK4a expression, and cellular growth in the ALCLs. To test whether NPM-ALK expression is required to maintain high levels of $p 16 I N K 4 a$ expression, we silenced NPMALK by RNA interference in the p16INK4a-expressing SU-DHL-1 ALCL cell line. As shown in Figure 4B, silencing of NPM-ALK resulted in decreased levels of p16INK4a mRNA and protein. To test whether loss of p16INK4a expression is critical for tumor growth in the p16INK4a-negative ALCLs, we re-expressed p16INK4a in the Karpas299 and DEL ALCL cell lines. Reexpression of p16INK4a in these cells impaired cell-cycle progression and, accordingly, reduced levels of phosphorylated $\mathrm{pRb}$ (Figure 4C). Notably, the same effect was obtained by treating the cells with the de-methylating agent 5-azacytidine, which induced the re-expression of p16INK4a (Figure 4D). 
A

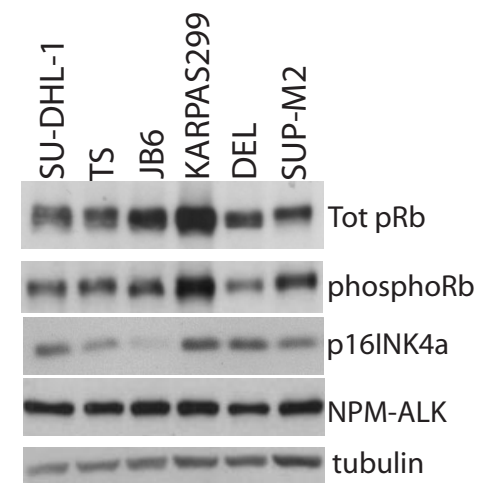

B

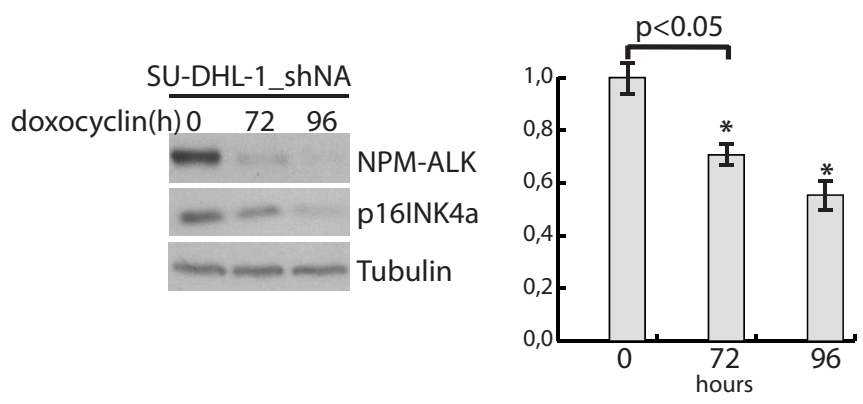

C

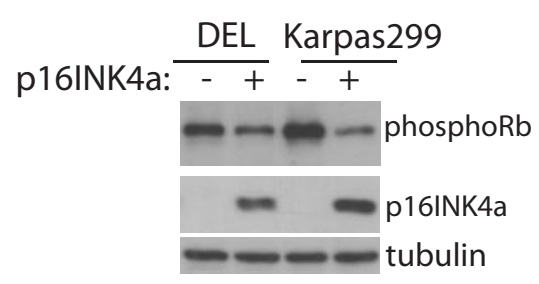

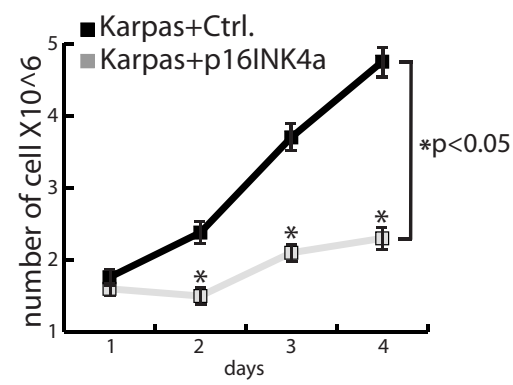

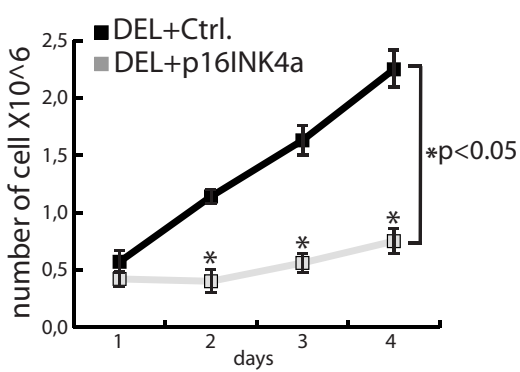

D
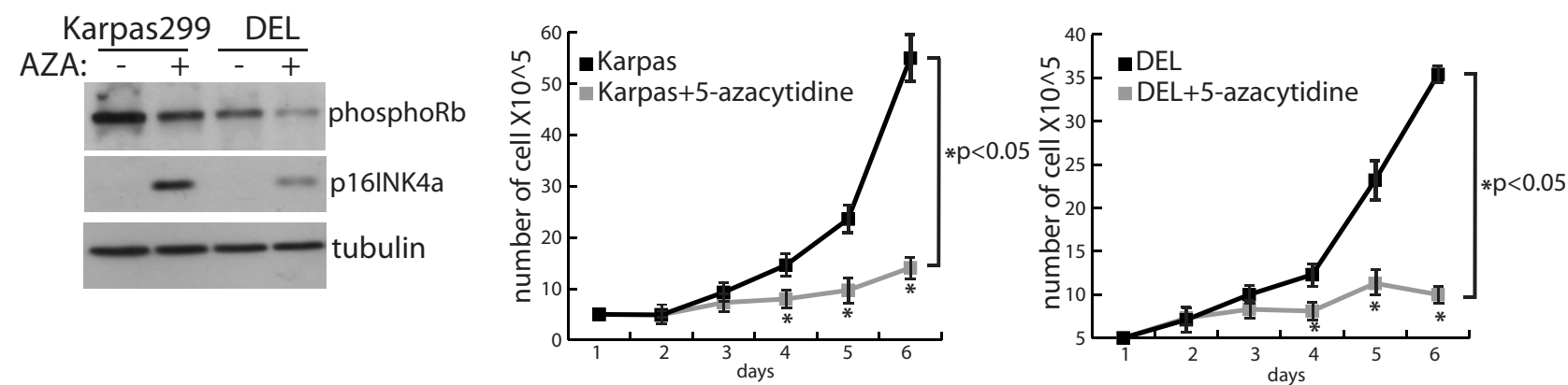

Figure 4. p16INK4a reactivation limits growth of ALCL cell lines. (A) Western blotting analysis of pRb, p16INK4a, phosphorylated pRb (serine 807-811), NPM-ALK, and tubulin expression in 6 different ALCL cell lines (as indicated). (B) Left panel: Western blot analysis of NPM-ALK, p16INK4a, and tubulin expression at different times (as indicated) in SU-DHL-1 cells treated with doxocyclin to induce shRNA interference of NPM-ALK. Right panel: p16INK4a mRNA quantification by qPCR analysis with specific primers. Data were standardized with ribosomal RNA and normalized against control untreated cells. (C) Karpas299 and DEL cell lines were infected with control (Ctrl) and p16INK4a-expressing retroviruses. Left panel: at day 4, cells were collected and expression of p16INK4a, phosphoRb, and tubulin evaluated by Western blot. Right panels: infected cells were selected, plated, and counted daily. (D) Karpas299 and DEL cell lines were treated with 5-azacytidine (AZA) for 6 days. Left panel: at day 5, cells were collected and expression of p16INK4a, phosphoRb, and tubulin evaluated by Western blot. Right panels: cells were plated and counted daily.

Finally, we investigated the molecular mechanisms underlying the effect of NPM-ALK on p16INK4a expression. p16INK4a transcription is repressed by polycomb-containing complexes, which induce tri-methylation of lysine 27 of histone H3 (H3K27 3 me) at the p16INK4a proximal promoter. Removal of $\mathrm{H} 3 \mathrm{~K} 27_{3 \mathrm{me}}$ is critical for $p 16 I N K 4 a$ transcriptional up-regulation during replicative senescence. ${ }^{26}$ We first investigated whether up-regulation of p16INK4a by NPM-ALK is associated with H3K27 de-methylation and increased pl6INK4a transcription, using chromatin immunoprecipitation (ChIP) and qPCR, respectively. NPM-ALK

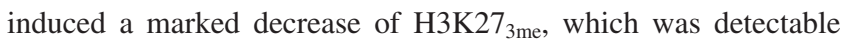
1 day after expression, and was more pronounced at days 3 and 5 (Figure 5A). Levels of p16INK4a transcripts also increased following NPM-ALK expression, with a slightly delayed kinetics (Figure $5 \mathrm{~A}$ ), suggesting that the effect of the fusion-protein on p16INK4a is transcriptional and involves de-methylation of H3K27.
We then examined NPM-ALK-dependent signaling pathways involved in p16INK4a transcriptional de-repression. The transcription factor JunB is overexpressed in human ALCLs and induces p16INK4a-dependent senescence in MEFs ${ }^{27}$ Expression of NPMALK in MEFs induced up-regulation of JunB (Figure 5C). However, NPM-ALK also induced p16INK4a up-regulation and proliferative arrest in MEFs in which JunB was silenced by short interfering RNA (shRNA), indicating that JunB is not involved in this process (Figure 5B-C). It has been recently shown that the histone de-methylase Jmjd3 actively de-methylates the p16INK4a promoter during oncogene- and stress-induced senescence, thus favoring p16INK4a transcriptional activation. ${ }^{28}$ In MEFs, NPMALK induced up-regulation of both Jmjd3 and p16INK4a (Figure 5D). Strikingly, NPM-ALK did not induce a proliferative arrest of Jmjd3-null MEFs, although cell growth of NPM-ALK-expressing Jmjd3-null MEFs was consistently lower than control Jmjd3-null 

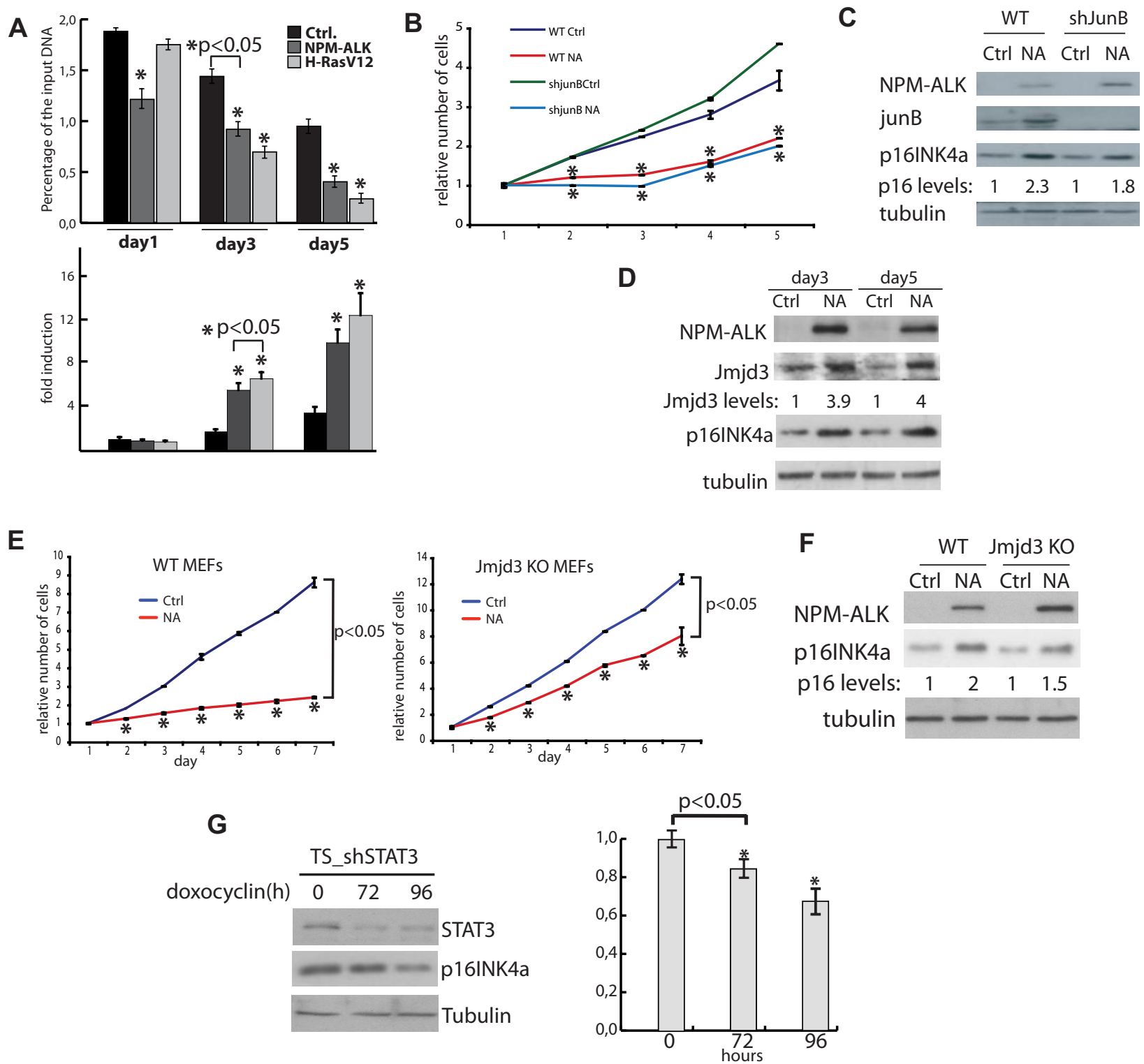

Figure 5. NPM-ALK induces p16INK4a promoter de-methylation. (A) Top panel: chromatin immunoprecipitation analysis of $p 16 I N K 4 a$ promoter H3K27me3 levels in WT MEFs infected with the indicated retroviruses. Precipitated DNA was amplified by qPCR using primers located in the p16INK4a promoter region. Enrichments are presented as percentage of total input DNA. Bottom panel: Q-PCR analysis of $p 16 I N K 4 a$ mRNA levels in the same cells, expressed as fold increase with respect to the day 1 control vector. (B) Growth curves of WT MEFs infected with a control lentivirus or lentiviruses expressing JunB-specific shRNAs, in the presence or absence of NPM-ALK, as indicated. Values are expressed relative to day 1 for each sample. ${ }^{*} P<.05$ with respect to control cells infected with a control vector. (C) Western blotting of p16INK4a, JunB, and NPM-ALK expression in the same cell samples as in panel B, 4 days after infection. Tubulin was used as a loading control. p16INK4a protein levels were calculated by densimetric analysis and normalized to control (Ctrl.) and tubulin band intensities. (D) Western blotting of Jmjd3 and p16INK4a expression levels in WT MEFs infected with NPM-ALK or control viruses. p16INK4a protein levels were calculated as in panel C. (E) Growth curves of WT and Jmjd3 null MEFs infected as indicated. Values are expressed relative to day 1 for each sample. (F) Western blotting of p16INK4a and NPM-ALK expression levels in WT and Jmjd3-null MEFs in the same cell samples as in panel E, 4 days after infection. p16INK4a protein levels were calculated as in panel C. (G) Left panel: Western blot analysis of STAT3, p16INK4a, and tubulin expression at different times (as indicated) in TS cells treated with doxocyclin to induce shRNA interference targeting STAT3. Right panel: p16INK4a mRNA quantification by qPCR analysis with specific primers. Data were standardized with ribosomal RNA and normalized against control untreated cells.

cells (Figure 5E). Accordingly, the NPM-ALK-induced p16INK4a up-regulation in Jmjd3-null MEFs was reduced, but not prevented (Figure 5F), suggesting that Jmjd3 only partially contributes to activation of p16INK4a by NPM-ALK. To investigate other pathways, we analyzed STAT3, a key transcription factor in the NPM-ALK signaling pathway. ${ }^{29}$ Down-regulation of STAT3 expression in the TS ALCL cell line by RNA interference, led to reduced levels of p16INK4a both at protein and mRNA level (Figure 5G). Thus, multiple signaling pathways, including Jmjd3 and STAT3, contribute to the activation of the p16INK4a tumor-suppressive pathway by NPM-ALK.

\section{Discussion}

Our data provide genetic evidence that the $\mathrm{p} 16 \mathrm{INK} 4 \mathrm{a} / \mathrm{pRb}$ pathway is involved in the execution of OIS, both in vitro and in vivo, and strongly suggest that it represents an alternative barrier to tumor development when tumorigenesis is initiated by oncogene mutations that inhibit the ARF/p53 signaling pathway. This conclusion is based on the finding that NPM-ALK limits p53 transcriptional activity when expressed in primary cells, yet it induces senescence by activating the $\mathrm{p} 16 \mathrm{INK} 4 \mathrm{a} / \mathrm{pRb}$ pathway, both in cultured cells 
and in vivo. This hypothesis is also supported by the observations that NPM-ALK lymphomagenesis is accelerated in mice lacking p16INK4a expression, and that $\mathrm{p} 16 \mathrm{INK} 4 \mathrm{a}$ and/or $\mathrm{pRb}$ expression is frequently lost in primary ALCLs, whereas p53 is variably expressed (10 of 19 cases with $>10 \%$ p53-positive tumor cells; Table 1), but never mutated (13 of 13 cases). The $\mathrm{p} 16 \mathrm{INK} 4 \mathrm{a} / \mathrm{pRb}$ pathway might have a similar function in other tumor types characterized by infrequent mutations of $\mathrm{p} 53$ and an overt ARF/p53 inhibitory effect of the corresponding initiating oncogene. This is the case for AMLs, including those expressing PML-RAR $\alpha$, cytoplasmic NPM, or B-cell receptor ABL. The list might be longer, considering that other AML-associated fusion proteins, such as TEL-JAK2, TEL-PDGFR, and TEL-TRKC, inactivate p53 and induce p16INK4a and cell-cycle arrest on expression in MEFs (our unpublished results).

The finding that NPM-ALK or other leukemia oncogenes antagonize the $\mathrm{ARF} / \mathrm{p} 53$ pathway argues against its involvement as a tumor-suppressive mechanism for these oncogenes. Indeed, NPM-ALK markedly reduced the clonogenic activity and replication potential of Arf-null $\mathrm{lin}^{-}$cells, and induced lymphomas with the expected latency and incidence when expressed into Arf-null mice (supplemental Figure 4A-B). However, expression of NPM/ ALK in Arf-null MEFs did not induce expression of SA- $\beta$ galactosidase (not shown) and only provoked a transient cell-cycle arrest, as, after 6-8 days, the cells re-entered the cell cycle (supplemental Figure 4C-D), suggesting that expression of ARF is required for the pro-senescence effect of NPM-ALK in MEFs. Even though MEFs do not represent the physiologic cell of origin of ALCLs, these findings suggest that in specific cell types and/or physiologic settings, the integrity of the ARF/p53 pathway might be necessary for the tumor-suppressive function of $\mathrm{p} 16 \mathrm{INK} 4 \mathrm{a} / \mathrm{Rb}$. Interestingly, p53 mutations are occasionally observed in ALCLs and other hematopoietic tumors (including APLs and AMLs).

Our data have implications for the treatment of ALCLs and other hematopoietic tumors carrying a WT ARF/p53 pathway. Re-expression of p53 in tumors with mutated Tp53 activates the senescence or apoptotic program and induces tumor regression. ${ }^{30}$ Under these circumstances, the exogenously expressed p53 becomes transcriptionally competent, because of the persistence of the mutated oncogene that ensures chronic activation of upstream components of the ARF/p53 pathway. The same effect can also be achieved with drugs that target p53 in tumors carrying WT Tp53 alleles and attenuated p53 signaling, because of genetic alterations of other components of the ARF/p53 pathway. ${ }^{31}$ In principle, this effect cannot be achieved in ALCLs or other hematopoietic tumors, where the ARF/p53 pathway, although unmutated, is functionally suppressed by NPM/ALK or other initiating oncogenes. Restoration of oncogene-induced senescence, in these tumors, might instead be achieved by reactivation of $\mathrm{p} 16 \mathrm{INK} 4 \mathrm{a} / \mathrm{pRb}$. Notably, our finding that 5-azacytidine-treatment of ALCL cells, in which pl6INK $4 a$ is inactivated by promoter hypermethylation, resulted in cell-cycle arrest, strongly suggests that specific de-methylating agents might find a role in the treatment of a subset of ALCLs.

\section{Acknowledgments}

We thank Paola Dalton for editing the manuscript. We thank Gioacchino Natoli for providing anti Jmjd3 antibody and Jmjd3 null MEFs.

This work was supported by grants from AIRC, EC, and Ministero della Salute to P.G.P. and E.C.

\section{Authorship}

Contribution: P.M., C.S., and P.B. performed most of the experiments and wrote the manuscript; G.P. and P.R.R. performed immunohistochemistry experiments on patient specimens; C.F. performed pl6INK4a promoter methylation analysis; S.V. performed p53 gene sequence analysis; R.C. and G.I. provided the NPM-ALK mouse models and critically revised the manuscript; S.P. provided some of the human specimens; B.K.T. provided Jmjd3 $3^{-1-}$ MEFs; S.D.T. and F.K.E.M. provided pRb phosphorylation data and prepared the manuscript; and P.G.P. and E.C. designed the study, supervised the experimental work, and wrote and critically revised the manuscript.

Conflict-of-interest disclosure: The authors declare no competing financial interests.

The current affiliation for P.M. is Centro Nacional de Investigaciones Oncológicas (CNIO), Madrid, Spain. The current affiliation for P.B. is Istituto Oncologico della Svizzera Italiana (IOSI), Bellinzona, Switzerland.

Correspondence: E. Colombo or P. G. Pelicci, Department of Experimental Oncology, Istituto Europeo di Oncologia, Via Adamello 16, 20139, Milano, Italy; e-mail: emanuela.colombo@ ifom-ieo-campus.it or piergiuseppe.pelicci@ifom-ieo-campus.it.

\section{References}

1. Collado M, Blasco MA, Serrano M. Cellular senescence in cancer and aging. Cell. 2007;130(2): 223-233.

2. Collado M, Serrano M. The power and the prom ise of oncogene-induced senescence markers. Nat Rev Cancer. 2006;6(6):472-476.

3. Serrano M, Lin AW, McCurrach ME, Beach D, Lowe SW. Oncogenic ras provokes premature cell senescence associated with accumulation of p53 and p16INK4a. Cell. 1997;88(5):593-602.

4. Kamijo T, Zindy F, Roussel MF, et al. Tumor suppression at the mouse INK4a locus mediated by the alternative reading frame product p19ARF. Cell. 1997;91(5):649-659.

5. Sharpless NE, Bardeesy N, Lee KH, et al. Loss of p16Ink4a with retention of p19Arf predisposes mice to tumorigenesis. Nature. 2001;413(6851): 86-91.

6. Sage J, Mulligan GJ, Attardi LD, et al. Targeted disruption of the three Rb-related genes leads to loss of $\mathrm{G}(1)$ control and immortalization. Genes Dev. 2000;14(23):3037-3050.

7. Halazonetis TD, Gorgoulis VG, Bartek J. An oncogene-induced DNA damage model for cancer development. Science. 2008;319(5868):13521355.

8. Insinga A, Monestiroli S, Ronzoni S, et al. Impairment of $p 53$ acetylation, stability and function by an oncogenic transcription factor. EMBO J.2004; 23(5):1144-1154.

9. Trotta R, Vignudelli T, Candini O, et al. BCR/ABL activates mdm2 mRNA translation via the La antigen. Cancer Cell. 2003;3(2):145-160.

10. Cui YX, Kerby A, McDuff FK, Ye H, Turner SD. NPM-ALK inhibits the p53 tumor suppressor pathway in an MDM2 and JNK-dependent manner. Blood. 2009;113(21):5217-5227.

11. Colombo E, Martinelli P, Zamponi R, et al. Delocalization and destabilization of the Arf tumor suppressor by the leukemia-associated NPM mutant. Cancer Res. 2006;66(6):3044-3050.

12. Amin HM, Lai R. Pathobiology of ALK+ anaplas tic large-cell lymphoma. Blood. 2007;110(7): 2259-2267.

13. Morris SW, Kirstein MN, Valentine MB, et al. Fusion of a kinase gene, ALK, to a nucleolar protein gene, NPM, in non-Hodgkin's lymphoma. Science. 1994;263(5151):1281-1284.

14. Chiarle R, Gong JZ, Guasparri I, et al. NPM-ALK transgenic mice spontaneously develop T-cell lymphomas and plasma cell tumors. Blood. 2003; 101(5):1919-1927.

15. Rassidakis GZ, Thomaides A, Wang S, et al. p53 gene mutations are uncommon but p53 is commonly expressed in anaplastic large-cell lymphoma. Leukemia. 2005;19(9):1663-1669.

16. Minucci S, Monestiroli S, Giavara S, et al. PMLRAR induces promyelocytic leukemias with high efficiency following retroviral gene transfer into 
purified murine hematopoietic progenitors. Blood. 2002;100(8):2989-2995.

17. Ventura A, Meissner A, Dillon CP, et al. Cre-loxregulated conditional RNA interference from transgenes. Proc Natl Acad Sci U S A. 2004; 101(28):10380-10385.

18. Piva R, Pellegrino E, Mattioli M, et al. Functional validation of the anaplastic lymphoma kinase signature identifies CEBPB and BCL2A1 as critical target genes. J Clin Invest. 2006;116(12):31713182.

19. Krimpenfort $P$, Quon KC, Mooi WJ, Loonstra A, Berns A. Loss of p16Ink4a confers susceptibility to metastatic melanoma in mice. Nature. 2001; 413(6851):83-86.

20. Colombo E, Marine JC, Danovi D, Falini B, Pelicci PG. Nucleophosmin regulates the stability and transcriptional activity of p53. Nat Cell Biol. 2002; 4(7):529-533.

21. Dimri GP, Lee $X$, Basile $G$, et al. A biomarker that identifies senescent human cells in culture and in aging skin in vivo. Proc Natl Acad Sci U S A. 1995;92(20):9363-9367.

22. Bonetti P, Davoli T, Sironi C, Amati B, Pelicci PG, Colombo E. Nucleophosmin and its AML-associated mutant regulate c-Myc turnover through Fbw7 gamma. J Cell Biol. 2008;182(1):19-26.

23. Herman JG, Graff JR, Myohanen S, Nelkin BD, Baylin SB. Methylation-specific PCR: a novel PCR assay for methylation status of $\mathrm{CpG}$ islands. Proc Natl Acad Sci U S A. 1996;93(18):9821-9826.

24. Kuefer MU, Look AT, Pulford K, et al. Retrovirus-mediated gene transfer of NPM-ALK causes lymphoid malignancy in mice. Blood. 1997;90(8):2901-2910.

25. Rassidakis GZ, Lai R, Herling M, Cromwell C Schmitt-Graeff A, Medeiros LJ. Retinoblastoma protein is frequently absent or phosphorylated in anaplastic large-cell lymphoma. Am J Pathol. 2004;164(6):2259-2267.

26. Bracken AP, Kleine-Kohlbrecher D, Dietrich N, et al. The Polycomb group proteins bind throughout the INK4A-ARF locus and are disassociated in senescent cells. Genes Dev. 2007;21(5):525-530.

27. Passegue E, Wagner EF. JunB suppresses cell proliferation by transcriptional activation of p16(INK4a) expression. EMBO J. 2000;19(12): 2969-2979.

28. Agger K, Cloos PA, Rudkjaer L, et al. The H3K27me3 demethylase JMJD3 contributes to the activation of the INK4A-ARF locus in response to oncogene- and stress-induced senescence. Genes Dev. 2009;23(10):1171-1176.

29. Zamo A, Chiarle R, Piva R, et al. Anaplastic lymphoma kinase (ALK) activates Stat3 and protects hematopoietic cells from cell death. Oncogene. 2002;21(7):1038-1047.

30. Kastan MB. Wild-type p53: tumors can't stand it. Cell. 2007;128(5):837-840.

31. Cicalese A, Bonizzi G, Pasi CE, et al. The tumor suppressor p53 regulates polarity of self-renewing divisions in mammary stem cells. Cell. 2009; 138(6):1083-1095 


\section{The lymphoma-associated NPM-ALK oncogene elicits a p16INK4a/pRb-dependent tumor-suppressive pathway}

Paola Martinelli, Paola Bonetti, Cristina Sironi, Giancarlo Pruneri, Caterina Fumagalli, Paola Rafaniello Raviele, Sara Volorio, Stefano Pileri, Roberto Chiarle, Fiona Kate Elizabeth McDuff, Betsabeh Khoramian Tusi, Suzanne D. Turner, Giorgio Inghirami, Pier Giuseppe Pelicci and Emanuela Colombo

Updated information and services can be found at:

http://www.bloodjournal.org/content/117/24/6617.full.html

Articles on similar topics can be found in the following Blood collections

Lymphoid Neoplasia (2140 articles)

Information about reproducing this article in parts or in its entirety may be found online at:

http://www.bloodjournal.org/site/misc/rights.xhtml\#repub_requests

Information about ordering reprints may be found online at:

http://www.bloodjournal.org/site/misc/rights.xhtml\#reprints

Information about subscriptions and ASH membership may be found online at:

http://www.bloodjournal.org/site/subscriptions/index.xhtml

Blood (print ISSN 0006-4971, online ISSN 1528-0020), is published weekly by the American Society of Hematology, 2021 L St, NW, Suite 900, Washington DC 20036.

Copyright 2011 by The American Society of Hematology; all rights reserved. 